\title{
INDICES ANTROPOMÉTRICOS E ESTADO NUTRICIONAL DE ESCOLARES DE BAIXA RENDA DE UM MUNICÍPIO DO ESTADO DO RIO DE JANEIRO (BRASIL): UM ESTUDO PILOTO*
}

\author{
Luiz Antonio dos Anjos**
}

\begin{abstract}
ANJOS, L.A. dos Índices antropométricos e estado nutricional de escolares de baixa renda de um município do Estado do Rio de Janeiro (Brasil): um estudo piloto. Rev. Saúde públ., S. Paulo, 23: $221-9,1989$.

RESUMO: Estudou-se o crescimento e o estado nutricional, por meio de índices antropométricos, de 185 crianças ( 97 meninos e 88 meninas) em idade escolar (7,0-10,9 anos) de baixa renda familiar do município de Nova Iguaçú, Estado do Rio de Janeiro (Brasil). A antropometria nutricional identificou 3,52 e $6,25 \%$ das crianças como desnutridas recentes e crônicas, respectivamente; valores que se comparam aos descritos para crianças faveladas do Município do Rio de Janeiro. Em geral, as medianas de altura das crianças ficaram abaixo do $25^{\circ}$ centil do padrão internacional de crescimento, sendo que a partir dos 10 anos a mediana da altura dos meninos foi inferior ao $10^{\circ}$ centil. As médias de peso e altura dessas crianças foram comparáveis à do nordeste urbano, superiores à da Paraiba, e inferiores às crianças de classe média de São Paulo. Os valores de 7 dobras cutâneas, do perímetro do braço, e da área de gordura do braço foram superiores nas meninas de todas as faixas etárias. A área muscular do braço foi maior nos meninos do que nas meninas de todas as faixas etárias.
\end{abstract}

DESCRITORES: Desnutrição protéico-calórica. Antropometria. Crescimento. Estado nutricional.

\section{INTRODUÇÃO}

Do ponto de vista de saúde pública, pesquisadores e clínicos estão interessados na avaliação do crescimento e estado nutricional de crianças até 6 anos, pois é essa a faixa etária crítica no tocante a mortalidade causada pela desnutrição protéico-energética. Os sobreviventes dessa fase enfrentarão alterações no crescimento tornando-se adultos com estatura menor. Com isso o ciclo de miséria se fecha e se perpetua pelos seus descendentes, pois uma criança desnutrida terá sua capacidade produtiva reduzida quando adulto $^{18}$. No Brasil existe uma carência de estudos em crianças em idade escolar e principalmente em populaçðes de alto risco, como as que vivem na periferia das grandes cidades brasileiras. O presente trabalho apresenta dados de índices antropométricos e estado nutricional de 185 crianças em idade escolar $(7,0$ a 10,9 anos de idade) de baixa renda familiar, freqüentadoras de uma escola da rede pública do Município de Nova Iguaçú, Estado do Rio de Janeiro.

\section{MATERIAL E MÉTODO}

As crianças desse estudo são de uma escola pública localizada na Chatúba, Baixada Fluminense, Município de Nova Iguaçú, Estado do Rio de Janeiro. Somente crianças que tinham uma cópia da certidão de nascimento nos arquivos da escola foram incluídas no estudo. A data de nascimento de cada criança foi copiada da certidão de nascimento e a idade em decimais foi calculada com precisão até o dia da coleta de dados. Foram examinadas todas as crianças disponiveis com idade entre 7,0 e 10,9 anos. Um total de 185 crianças ( 97 meninos e 88 meninas) foram incluídas no trabalho. Dezoito porcento das meninas e $19 \%$ dos meninos eram brancos, $30 \%$ das meninas e $41 \%$ dos meninos eram negros, sendo $52 \%$ das meninas e $40 \%$ dos meninos mulatos. Um questionário socio-econômico preenchido por meio de entrevista com os pais indicou que a renda familiar média dessas crianças era em torno de um salário mínimo regional'. Durante essa entrevista a

\footnotetext{
* Parte desse trabalho foi apresentado na $40^{\text {a }}$ Reunião anual da Socıedade Brasileira para o Progresso da Ciência, julho, 1988.

** Department of Kinesiology, University of Illinois, $906 \mathrm{~S}$. Goodwin Ave., Urbana, Illinois 61801, USA. Bolsista da Coordenadoria de Aperfeiçoamento do Pessoal do Ensino Superior (CAPES), Brasília, Brasil. Proc. no 3612/85-3.
} 
autorização verbal dos pais para a participação das crianças no estudo foi obtida.

Todas as medidas foram feitas na escola durante horário escolar pelo mesmo investigador. $O$ peso foi medido seguindo padronização do Programa Biológico Internacional ${ }^{21}$ em balança médica (Filizola), com precisão até $100 \mathrm{~g}$, com as crianças descalças e usando o mínimo possível de roupas. Antes de cada seção de coleta de dados a balança foi calibrada. A altura foi medida com precisão até décimos do centímetro com a criança em apnéia respiratória, em posição ortostática, com os pés juntos, com as mãos nos quadris e com a cabeça mantida no plano de Frankfurt. Os dados de peso e altura de cada criança foram comparados com o padrão de peso e altura americano compilado e publicado pelo National Center for Health and Statistics $\left(\mathrm{NCHS}^{10}\right)$. Os valores de " $\mathrm{Z}$ " para os índices antropométricos, peso para altura e altura para idade, foram obtidos para cada criança, por meio de programa para microcomputador desenvolvido pelo "Center for Disease Control", em Atlanta, EUA.

O perímetro do braço direito foi medido horizontalmente no meio da distância entre o processo acromial e o cotovelo com a criança em posição ortostática com os braços relaxados ao longo do corpo. As medidas (três) foram feitas com uma fita Lufkin, e a média usada para análise estatística. As dobras cutâneas (DC) do lado direito do corpo (bíceps, tríceps, subscapular, axilar média, suprailíaca, abdominal e panturrilha) foram medidas com um compasso Lange em milímetros, seguindo padronização de localização de Lohman e col. ${ }^{12}$. Para a DC do tríceps a prega cutânea foi levantada $1 \mathrm{~cm}$ acima do ponto médio da distância entre os processos acromial e olecraniano (onde a medida foi feita) na região posterior do braço. A DC do bíceps foi feita no mesmo lugar da do tríceps, mas na parte anterior do braço. Para a DC subescapular a prega cutânea foi levantada obliquamente $1 \mathrm{~cm}$ abaixo do ângulo inferior da escápula. A DC axilar média foi medida obliquamente na intersecção da linha axilar média vertical e uma linha horizontal que passa pelo apêndice xifóide. Na DC suprailíaca a prega cutânea foi levantada $2 \mathrm{~cm}$ acima da crista ilíaca ao nível da linha axilar média vertical; a medida foi feita $1 \mathrm{~cm}$ acima da crista ilíaca. $\mathrm{Na}$ DC abdominal a prega cutânea foi levantada 2 $\mathrm{cm}$ ao lado da cicatriz umbelical com o compasso sendo colocado verticalmente $1 \mathrm{~cm}$ abaixo da prega levantada. Para a medida da DC da panturrilha, o pé direito foi colocado num banco com o joelho levemente fletido. A medida foi feita verticalmente na maior circunferência da panturrilha. As medidas de dobras cutâneas foram feitas em todos os lugares cada uma das três vezes seguindo a mesma ordem todas as vo zes, com a média sendo usada na análise estatística. A média das 3 medidas das $7 D C\left(\sum 7 D C\right)$ foram somadas e comparadas entre sexo em cada faixa etária. Com os dados de perímetro do braço (PERI) e DC tríceps (TRI) as áreas do braço, muscular do braço e de gordura do braço foram estimadas segundo as fórmulas publicadas por Frisancho ${ }^{6}$ que são: área do braço = $(\pi / 4)$. (PERI $\left./ \pi^{2}\right)$; área muscular do braço $=$ $(\text { PERI }-\pi . T R I)^{2} / 4 \pi$; e área de gordura $=$ área do braço -área muscular do braço.

A análise estatística foi feita usando-se pacote estatístico ( $\mathrm{SAS}^{17}$ ) num computador central na Universidade de Illinois. As médias dos valores de peso, altura, DC, $\Sigma 7$ DC, PERI, áreas do braço, e valores " $Z$ " dos índices peso para altura e altura para idade foram comparadas entre os meninos e as meninas de cada faixa etária usando-se o teste " $t$ " de Student para medidas não pareadas. O valor de alfa igual a 0,05 foi escolhido para determinar significância na diferença entre as médias. Na reprodutibilidade das medidas o coeficiente de correlação "Pearson" foi calculado entre a primeira e a segunda avaliação. As médias da primeira e da segunda avaliações de cada variável foram comparadas usando-se o teste " $t$ " de Student para medidas pareadas.

\section{REPRODUTIBILIDADE DAS MEDIDAS}

Num intervalo de uma semana, 27 crianças ( \pm $15 \%$ da amostra) recrutadas aleatoriamente foram reavaliadas usando a mesma metodologia. A relação de todos os coeficientes de correlação entre a primeira avaliação e a segunda avaliação, assim como as médias das duas avaliaçðes com o erro padrão da média e o valor " $t$ " da comparação entre as duas médias são apresentadas na Tabela 1. Não houve significância entre as médias das duas avaliaçðes de nenhuma variável. Os coeficientes de correlação "Pear. son" foram bastante altos, variando de 0,914 (dobra cutânea Suprailiaca) a 0,997 (altura) entre a primeira avaliação e a segunda avaliação, indicando um alto índice de reprodutibilidade na medição das variáveis estudadas.

\section{RESULTADOS}

As médias de peso e altura são apresentadas 
para meninos e meninas em função da faixa etária na Tabela 2. Não houve diferença significativa entre sexo em nenhuma faixa etária.

A distribuição dos valores " $Z$ " dos índices peso para altura e altura para idade são apresentados na Tabela 3. Esses indicadores são utilizados na avaliação nutricional de crianças até 10 anos $^{2,22}$, portanto os dados da Tabela 3 representam 145 crianças na faixa etária de 7,0 a 9,9 anos. Para o índice peso para altura, 3,52\% das crianças apresentaram valores " $Z$ " inferiores a -2 (desnutrição recente), enquanto $6,25 \%$ das crianças apresentaram valores " $Z$ ", inferiores a - 2 para o índice altura para idade (desnutrição crônica). Notar que para ambos os indices o grupo modal de valores ' $Z$ " foi de $\cdot 0,9 \cdot$ até 0 , e que 67,60 e $86,11 \%$ das crianças apresentaram valores $Z$ negativos (inferiores a mediana do padrão) para os índices peso para altura e altura para idade, respectivamente.

As médias e erros padrão da média dos valores " $Z$ " dos índices peso para altura e altura para idade em função da idade e sexo, são apresentadas na Tabela 4. A única diferença signifi- cativa entre sexo foi a altura para idade na faixa etária de 7,0 a 7,9 anos.

As medianas de peso e aitura em relação a idade são apresentadas graficamente com a mediana (50. centil) do padrão americano do $\mathrm{NCHS}^{10}$ nas Figuras 1 e 2 . A mediana da altura das meninas da faixa etária 8-8,9 anos ficou entre o 25. e o $50^{\circ}$ centil do padrão NCHS. As medianas da altura dos meninos e das meninas das outras faixas etárias ficaram abaixo do 25 ? centil, sendo que a mediana da altura dos meninos de 10 anos ficou abaixo do 10 . centil.

As médias e desvios padrão das dobras cutâneas são apresentadas nas Tabelas 5 e 6 para os meninos e as meninas, respectivamente, em função da idade. Os valores para os meninos tenderam a crescer dos 7 para os 8 anos e a diminuir daí em diante. Já para as meninas a tendência foi dos valores aumentarem com a idade. Os valores individuais de cada lugar de dobra cutânea e o somatório das 7 dobras foram significativamente maiores $(\mathrm{p}<0,01)$ nas meninas do que nos meninos para todas as idades, com exceção nas crianças de 8 anos.

\section{TABELA 1}

Reprodutibilidade das medidas antropométricas.

\begin{tabular}{|c|c|c|c|c|c|c|c|}
\hline \multirow{2}{*}{ Vanáveis } & \multicolumn{3}{|c|}{$\begin{array}{l}\text { Primeira } \\
\text { Medida }\end{array}$} & \multicolumn{2}{|c|}{$\begin{array}{l}\text { Segunda } \\
\text { Medida }\end{array}$} & \multirow{2}{*}{$\mathrm{t}$} & \multirow{2}{*}{$r$} \\
\hline & $\mathrm{n}$ & Média & EPM* & Média & EPM* & & \\
\hline Peso $(\mathrm{kg})$ & 27 & 25,6 & 0,74 & 25.5 & 0,75 & 0,04 & 0,987 \\
\hline Altura $(\mathrm{cm})$ & 27 & 128,9 & 1,46 & 129,0 & 1,48 & $-0,02$ & 0,997 \\
\hline Perímetro do Braço $(\mathrm{cm})$ & 27 & 17,5 & 0,26 & 17,4 & 0,26 & 0,18 & 0,983 \\
\hline \multicolumn{8}{|l|}{ Dobras Cutâneas (mm): } \\
\hline Bíceps & 27 & 4,8 & 0,29 & 4,7 & 0,32 & 0,16 & 0,970 \\
\hline Tríceps & 27 & 8,8 & 0,51 & 8,5 & 0,45 & 0,38 & 0,973 \\
\hline Subscapular & 27 & 59 & 0,40 & 5,8 & 0,36 & 0,17 & 0,966 \\
\hline Axilar Média & 27 & 4,5 & 0,33 & 4,6 & 0,35 & $-0,15$ & 0,983 \\
\hline Supra Ilíaca & 27 & 4,7 & 0,40 & 4,9 & 0,49 & $-0,30$ & 0,914 \\
\hline Abdominal & 27 & 6.7 & 0,64 & 6,6 & 0,59 & 0,13 & 0,978 \\
\hline Panturrilha & 27 & 9,1 & 0,56 & 8,9 & 0,55 & 0,22 & 0,970 \\
\hline
\end{tabular}

* Os valores são médias e erro padrão da média (EPM) da primeira e segunda medida, o valor de $t$ para a comparação das médias do teste de $t$ de "Student" para medidas pareadas e o coeficiente de correlação (r) entre a primeira e a segunda medida.

\section{TABELA 2}

Valores de peso e altura de 185 escolares de 7,0 a 10,9 anos de idade, de Nova Iguaçú, RJ,

\begin{tabular}{|c|c|c|c|c|c|c|}
\hline \multirow{2}{*}{$\begin{array}{l}\text { Faixa } \\
\text { Etária }\end{array}$} & \multicolumn{4}{|c|}{ Peso $(\mathrm{kg})$} & \multicolumn{2}{|c|}{ Altura $(\mathrm{cm})$} \\
\hline & $\mathrm{n}$ & Meninas & $\mathrm{n}$ & Meninos & Meninas & Meninos \\
\hline $\begin{array}{c}7,0-7,9 \\
8,0-8,9 \\
9,0-9,9 \\
10,0-10,9\end{array}$ & $\begin{array}{l}19 \\
28 \\
29 \\
21\end{array}$ & $\begin{array}{l}21,7 \pm 2,7 \\
24,8 \pm 4,6 \\
26,0 \pm 3,1 \\
27,5 \pm 2,5\end{array}$ & $\begin{array}{l}21 \\
29 \\
19 \\
19\end{array}$ & $\begin{array}{l}22,7 \pm 3,1 \\
23,6 \pm 2,6 \\
26,3 \pm 4,3 \\
29,0 \pm 3,7\end{array}$ & $\begin{array}{l}119,6 \pm 5,8 \\
126,6 \pm 5,9 \\
130,4 \pm 5,4 \\
133,4 \pm 5,8\end{array}$ & $\begin{array}{l}122,8 \pm 6,4 \\
123,9 \pm 3,8 \\
129,7 \pm 5,3 \\
133,8 \pm 8,9\end{array}$ \\
\hline
\end{tabular}

* Valores são médias \pm desvio padrão. 
TABELA 3

Valores " $\mathrm{Z}$ " (em números absolutos e (\%)) dos índices antropométricos peso para altura e altura para idade em relação ao padrão americano NCHS 10 em 145 escolares de 7,0 a 9,9 anos de idade de Nova Iguaçú, RJ.

\begin{tabular}{ccccc}
\hline & \multicolumn{4}{c}{ Valores " $\mathrm{Z}$ " } \\
\cline { 2 - 5 } Variáveis & $\leqslant-2,0$ & $-1,9$ até $-1,0$ & $-0,9$ a té 0 & $\geqslant 0,1$ \\
\hline Peso para Altura & & & $60(42,25)$ & $46(32,40)$ \\
Altura para Idadeb & $5(3,52)$ & $31(21,83)$ & $72(50,00)$ & $20(13,89)$ \\
\hline
\end{tabular}

$\mathrm{a}_{\mathrm{n}}=142$

$b_{n}=144$

TABELA 4

Valores " $\mathrm{Z}$ " dos indices antropométricos peso para altura e altura para idade em relação ao padrão americano NCHS 10 , em função de sexo e faixa etária de 145 escolares de 7,0 a 9,9 anos de idade, de Nova Iguaçu, RJ*.

\begin{tabular}{cccccc}
\hline \multirow{2}{*}{ Faixa etária } & \multicolumn{2}{c}{ Peso para Altura } & & \multicolumn{2}{c}{ Altura para idade } \\
\cline { 2 - 3 } \cline { 5 - 6 } & Meninas & Meninos & & Meninas & Meninos \\
\hline $7,0-7,9$ & $-0,34 \pm 0,20$ & $-0,32 \pm 0,19$ & & $-0,31 \pm 0,18$ & $-0,88 \pm 0,18^{* 1}$ \\
$8,0-8,9$ & $-0,11 \pm 0,21$ & $-0,39 \pm 0,23$ & & $-0,77 \pm 0,12$ & $-0,64 \pm 0,20$ \\
$9,0-9,9$ & $-0,39 \pm 0,23$ & $-0,56 \pm 0,14$ & & $-0,84 \pm 0,20$ & $-0,94 \pm 0,13$ \\
\hline
\end{tabular}

Valores são médias \pm erro padrão da média.

' $\mathrm{p}=0,04$

TABELA 5

Valores de dobras cutâneas $(\mathrm{mm})$ de 97 escolares do sexo masculino, 7,0 a 10,9 anos de idade de Nova Iguaçú, RJ, por faixa etária*.

\begin{tabular}{lcccc}
\hline \multirow{2}{*}{ Dobras cutâneas } & \multicolumn{5}{c}{ Faixa etária } \\
\cline { 2 - 5 } & $7,0-7,9$ & $8,0-8,9$ & $9,0-9,9$ & $10,0-10,9$ \\
\hline $\mathrm{n}$ & 19 & 28 & 29 & 21 \\
Tríceps & $7,1 \pm 1,6$ & $8,8 \pm 4,4$ & $8,2 \pm 2,4$ & $7,4 \pm 1,7$ \\
Subescapular & $4,6 \pm 0,9$ & $6,5 \pm 5,1$ & $5,4 \pm 1,2$ & $5,1 \pm 1,0$ \\
Axilar média & $3,7 \pm 0,9$ & $5,1 \pm 4,1$ & $4,1 \pm 0,9$ & $3,8 \pm 0,8$ \\
Bíceps & $4,0 \pm 1,1$ & $5,1 \pm 3,3$ & $4,4 \pm 1,1$ & $4,1 \pm 1,0$ \\
Supra iliaca & $3,8 \pm 1,2$ & $5,3 \pm 4,4$ & $4,2 \pm 1,2$ & $4,1 \pm 1,1$ \\
Abdominal & $5,2 \pm 1,4$ & $8,1 \pm 7,1$ & $6,6 \pm 2,9$ & $6,3 \pm 2,0$ \\
Panturrilha & $7,0 \pm 2,0$ & $8,8 \pm 4,1$ & $7,9 \pm 2,4$ & $7,1 \pm 1,8$ \\
& & & $40,9 \pm 10,8$ & $38,0 \pm 8,0$ \\
\hline
\end{tabular}

- Valores são médias \pm desvio padrão. 


\section{TABELA 6}

Valores de dobras cutâneas (mm) de 88 escolares do sexo feminino, 7,0 a 10,9 anos de idade de Nova Iguaçú, RJ, por faixa etária*.

\begin{tabular}{lcccc}
\hline \multirow{2}{*}{ Dobras cutâneas } & \multicolumn{4}{c}{ Faixa etária } \\
\cline { 2 - 5 } & $7,0-7,9$ & $8,0-8,9$ & $9,0-9,9$ & $10,0-10,9$ \\
\hline $\mathrm{n}$ & 21 & 29 & 19 & 19 \\
Tríceps & $9,5 \pm 2,9$ & $9,8 \pm 2,4$ & $10,1 \pm 2,4$ & $10,1 \pm 2,5$ \\
Subescapular & $6,0 \pm 2,2$ & $6,6 \pm 2,4$ & $6,7 \pm 2,1$ & $7,3 \pm 2,4$ \\
Axilar média & $5,2 \pm 2,6$ & $5,0 \pm 2,0$ & $5,2 \pm 1,6$ & $5,6 \pm 1,9$ \\
Bícéps & $5,4 \pm 1,6$ & $5,4 \pm 1,6$ & $5,7 \pm 1,5$ & $6,0 \pm 1,8$ \\
Supra ilíaca & $5,6 \pm 3,0$ & $5,4 \pm 2,5$ & $5,6 \pm 2,0$ & $6,4 \pm 2,4$ \\
Abdominal & $7,7 \pm 4,2$ & $8,2 \pm 4,4$ & $8,1 \pm 3,2$ & $9,6 \pm 3,6$ \\
Panturrilha & $9,6 \pm 3,2$ & $10,2 \pm 2,7$ & $11,6 \pm 4,3$ & $11,5 \pm 3,4$ \\
& & & & $53,0 \pm 15,3$ \\
$\Sigma$ das 7 Dobras & $48,8 \pm 18,5$ & $50,6 \pm 16,5$ & $56,5 \pm 16,0$ \\
\hline
\end{tabular}

- Valores são médias \pm desvio padrão.

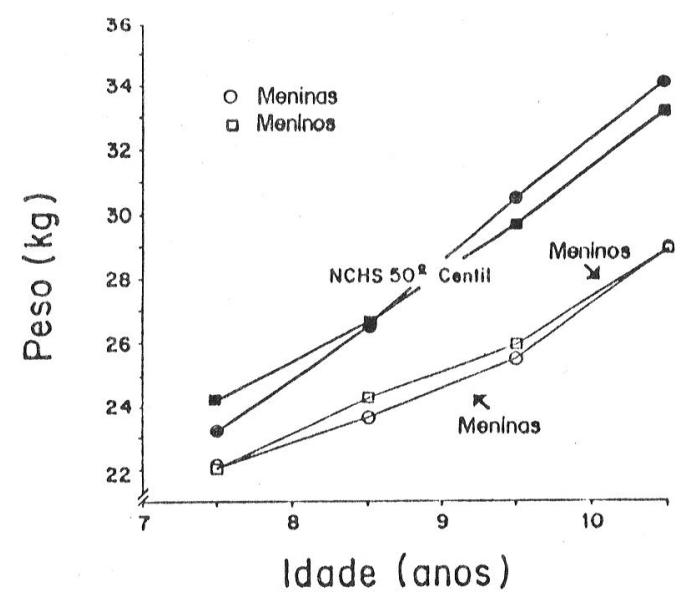

Fig. 1 - Medianas de peso em função da idade. A curva em negrito é a mediana (50. centil do padrão americano $\mathrm{NCHS}^{10}$ ).

Os valores médios de perímetro do braço e das áreas musculares e de gordura do braço são apresentados graficamente na Figura 3 (A, B e C) em função do sexo e faixa etária. O perímetro do braço foi maior nas meninas do que nos meninos de todas as idades, com exceção da idade de 8 anos. Entretanto, os meninos tenderam a ter valores superiores de área muscular do que as meninas em todas as idades. Esse fato se deve aos valores maiores de dobra cutânea

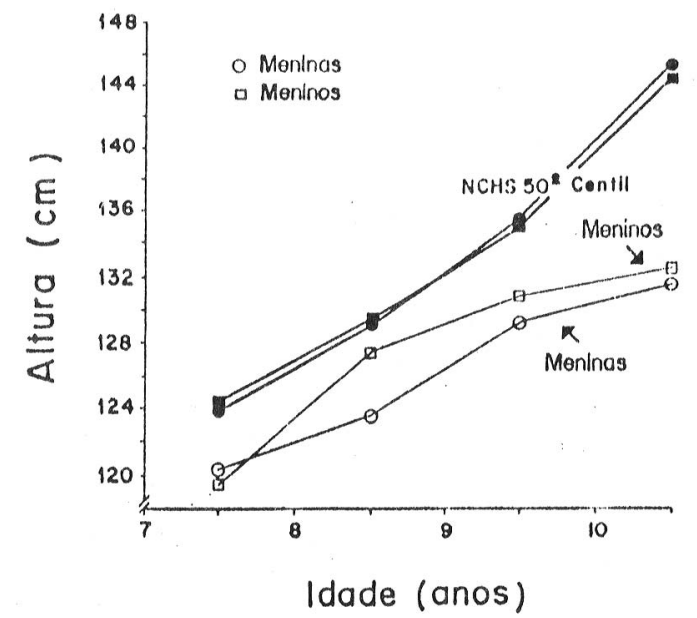

Fig. 2 - Medianas da altura em função da idade. A curva em negrito é a mediana ( $50^{\circ}$ centil do padrăo americano $\mathrm{NCHS}^{10}$ ).

nas meninas, o que faz com que a área de gordura do braço seja sistematicamente superior nas meninas de todas as idades (Figura $3 \mathrm{C}$ ). Como acima citado a curva de dobra cutânea dos meninos apresentou um padrão em "U' invertido, ou seja tenderam a crescer dos 7 para os 8 anos e a diminuirem daí em diante. Esse fato fez com que o mesmo ocorresse na curva de área de gordura do braço dos meninos (Figura 3C). 

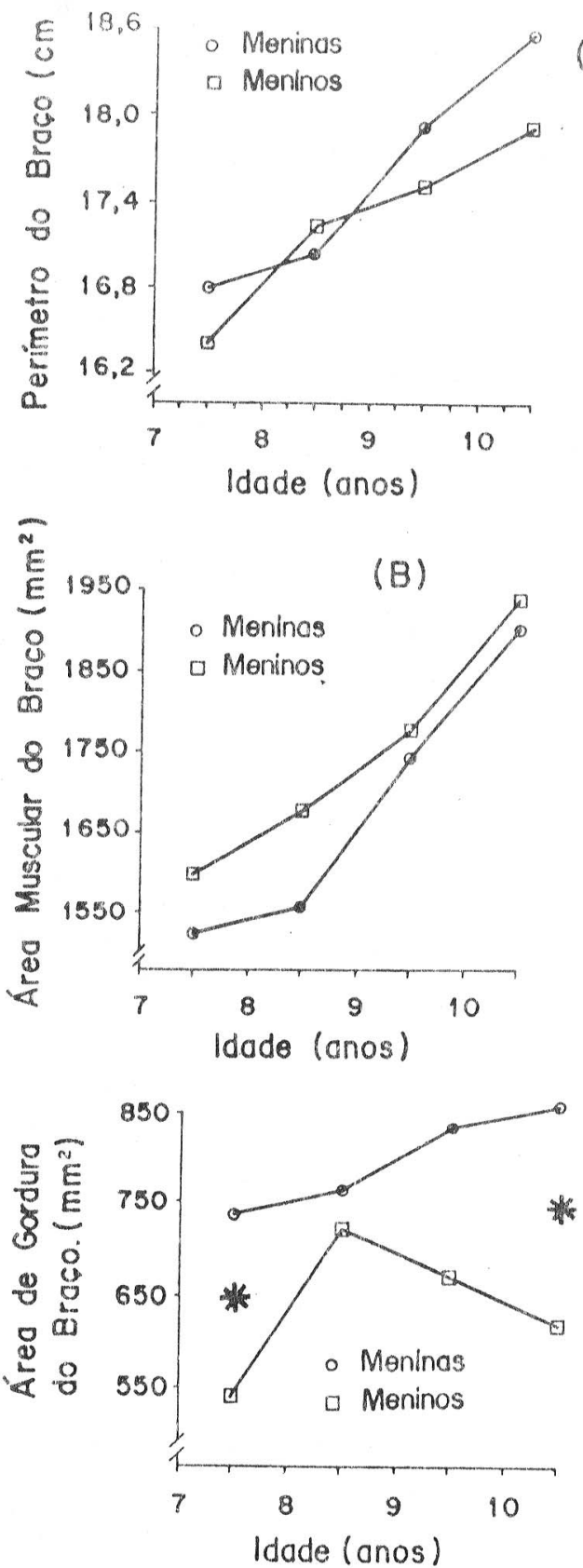

Fig. 3 - Gráficos das médias do perimetro do braço (A) e das áreas muscular (B) e de gordura (C) do braço em relação a idade e sexo. O asterisco mostra diferença significativa entre meninos e meninas ( $p$ $<0,05$ ) para a respectiva idade.

\section{DISCUSSÃO}

Os índices antropométricos em crianças servem para avaliar o crescimento dessas crianças e indiretamente para caracterizar o estado nutricional da população, ao que se tem chamado de antropometria nutricional ${ }^{2,16}$. As crianças do presente estudo moravam em condições precárias e freqüentavam a mesma escola pública.
Uma avaliação sócio-econômica com os pais evidenciou que a renda familiar era em torno de um salário mínimo regional', caracterizando uma população homogênea com baixa qualidade de vida, e portanto com provável comprometimento do estado nutricional.

Não houve diferença de peso e altura entre os meninos e as meninas de nenhuma faixa etária, fato internacionalmente documentado em crianças pré-puberes ${ }^{5,9}$. Os valores de peso e altura das crianças do presente estudo são, em média, inferiores $3 \mathrm{~kg} \mathrm{e} 3 \mathrm{~cm}$ para os meninos e $1,5 \mathrm{~kg}$ e $2 \mathrm{~cm}$ para as meninas da mesma idade, de classe média, da rede escolar pública do $\mathrm{Mu}$ nicípio de São Caetano do Sul, Estado de São Paulo, publicados por Soares e col. ${ }^{19}$. As médias de peso e altura das crianças de Nova Iguaçú parecem ser superiores aos das crianças do Estado da Paraíba, apresentados por Cariri Benigna e col. ${ }^{3}$, mas se comparam aos de crianças nordestinas moradoras em zonas metropolitanas $^{3}$. A diferença no crescimento de crianças de classes sócio-econômicas diferentes no mesmo país já foi bastante documentada internacionalmente $4,5,7,9,14,20$. Os valores médios de peso e altura desse estudo são comparáveis aos de crianças americanas de descendência mexicana, da cidade de Brownsville, Texas, EUA, publicados por Zavaleta e Malina ${ }^{25}$, o que poderia indicar que apesar das condiçðes ambientais serem possivelmente melhores naquela população, os fatores nutricionais de influência no retardo do crescimento dos dois grupos de crianças possam se assemelhar.

Utilizando-se a metodologia preconizada por Waterlow e col. ${ }^{23}$ foi possível identificar $3,52 \mathrm{e}$ $6,25 \%$ das crianças como desnutridas recentes e crônicas. A prevalência de desnutrição crônica compara-se com a encontrada por Gross e col. ${ }^{8}$ para crianças até 2 anos de idade, de uma favela do Município do Rio de Janeiro, que variou de 4,9 a $6,4 \%$ entre os anos de 1981 e 1983 . A prevalência de desnutrição recente nas crianças do presente estudo foi superior a encontrada nas crianças faveladas em todos os anos $(0,7$ a $2,5 \%$ ). Não é do conhecimento do autor nenhum outro estudo onde essa metodologia tenha sido usada em crianças pré-escolares do Rio de Janeiro. Num estudo longitudinal de quase 5.000 crianças, desde o nascimento até 52 meses, em Pelotas, Estado do Rio Grande do Sul, a prevalência de desnutrição crônica variou entre 5,3 e $12,2 \%$, enquanto a desnutrição recente variou de 0,3 a $1,3 \%$ dependendo da ida$\mathrm{de}^{22}$. Comparativamente, portanto, parece que a prevalência de desnutrição crônica nas crianças do presente estudo assemelha-se a de crian- 
ças até 2 anos de idade do Rio de Janeiro e de Pelotas.

Os valores médios " $Z$ " dos índices antropométricos peso para altura e altura para idade foram negativos para todas as faixas etárias em ambos os sexos, sendo que a única diferença significativa entre sexo aconteceu para altura para idade na faixa etária de 7,0-7,9 anos, aparentemente devido a um valor médio mais baixo para as meninas do que para os meninos (- 0,31 e - 0,88, respectivamente, Tabela 4), o que provavelmente não tem significado fisiológico.

A comparação da mediana de peso e principalmente da altura dessas crianças com o padrão americano mostra uma diferença acentuada entre as duas populações principalmente na altura das crianças da faixa etária 10,0-10,9 anos do presente estudo, cuja mediana ficou abaixo do 10 . centil do padrão americano. A possivel explicação para essa diferença é o nível de maturação. As crianças americanas de 10 anos já começaram a aceleração do crescimento da puberdade e nenhuma das crianças desse estudo apresentaram sinais clínicos de início da puberdade. Crianças que vivem sob estresse social e nutricional tendem a atrasar o início da maturação ${ }^{13}$, e daí a possivel explicação para a grande diferença de altura entre as duas populaçðes, principalmente a partir dos 10 anos de idade. E por este fato que se recomenda a utilização do padrão internacional somente em crianças até 10 anos de idade $e^{2,8,23,24}$.

Com exceção da dobra cutânea axilar média da faixa etária 8,0-8,9 anos, as meninas apresentaram valores superiores aos dos meninos em todas 7 dobras cutâneas (DC) estudadas. Os valores de dobras cutâneas das crianças do presente estudo são em média inferiores a 4 e $1 \mathrm{~mm}$ para os meninos e 5 e $2 \mathrm{~mm}$ para as meninas, respectivamente para as DC tricipital e subescapular em relação a crianças da mesma idade de classe média alta do Município de São Paulo, apresentados por Hegg". Não há explicações para o padrão de "U" invertido observado nos valores de dobras cutâneas dos meninos em relação a idade a não ser uma possível flutuação da amostra, pois não há explicação fisiológica para que isso ocorresse, muito embora um tipo de padrão serrilhado (sobe e desce) foi observado por Matsudo e col. ${ }^{15}$ em DC de crianças de 11 a 14 anos.

Apesar das meninas tenderem a ter valores maiores de perímetro do braço do que os meninos, os meninos sistematicamente tiveram área muscular do braço maiores do que as meninas (Figura 3A e B). Esse fato se deve aos valores da DC tricipital superiores nas meninas. A maior adiposidade em meninas do que em meninos da mesma idade é um fato bastante conhecido, mas foi interessante observar que esse fato ocorre mesmo em populaçðes de baixa renda.

Em suma, as crianças de baixa renda familiar do presente estudo apresentaram déficits importantes de crescimento em relação a padroes internacionais. A prevalência de desnutrição foi comparável a de um estudo numa favela do Rio de Janeiro ${ }^{8}$. As médias de peso e altura foram inferiores as de crianças da mesma idade de classe média de São Paulo" e superiores as de crianças do Estado da Paaíba ${ }^{3}$. Esses déficits deverão representar uma estatura adulta menor o que poderá acarretar deficiências na capacidade para o trabalho físico. As precárias condiçðes ambientais em que essas crianças vivem irão definitivamente prejudicar o. desenvolvimento físico e intelectual nessa população que rapidamente se multiplica nas áreas periféricas das grandes cidades no Brasil.

\section{AGRADECIMENTOS}

Às professoras Alda de Carvalho Fonseca e Jacqueline Schwarcfuter, diretoras da escola estudada, e aos demais professores da mesma escola, pelas facilidades oferecidas na fase de coleta de dados; à Wanda Ferro de Barros, à Sônia Regina Alves Couto e à Dra. Sandra Helena Couto Afonso pela colaboração na coleta de dados. 
ANJOS, L.A. dos [Anthropometric indices and nutritional status of low-income school-aged children of a county of the State of Rio de Janeiro, Brazil: a pilot study[. Rev. Saúde públ., S. Paulo, 23:221-9, 1989.

ABSTRACT: The growth and nutritional status of 185 school-aged children ( 97 boys and 88 girls) of low socio-economic level in Nova Iguaçu, State of Rio de Janeiro, Brazil, were studied. Nutritional anthropometry identified 3.52 and $6.25 \%$ of the children as suffering from wasting and stunting, respectively. These prevalences of malnutrition were comparable to those described in pre-school children living in a "favela" (shanty town) of Rio de Janeiro. In general, the median height fell below the $25^{\text {th }}$ centile of the international standard of growth. The value of 10 year-old boys fell below the $10^{\text {th }}$ centile. The mean values of weight and height of these children were comparable to those of children from the Northeastern region of Brazil ("Nordeste"), higher than those found for children in the State of Paraiba, Brazil, and lower than those for middle-class children of the State of S. Paulo. Skinfold thickness, arm circunference, and arm fat area data were higher in girls than boys. However, arm muscle are values in boys were superior in comparison to those of girls.

KEYWORDS: Protein-calorie malnutrition. Antropometry. Growth. Nutritional status.

\section{REFERENCIAS BIBLIOGRÁFICAS}

1. ANJOS, L.A. Relação entre índices antropométricos e fatores sociais em escolares da Baixada Fluminense, RJ. [Resumo], Cienc. e Cult., 40(supl.):103, 1988.

2. ANJOS, L.A. Antropometria nutricional: uso de dados de peso e altura na avaliação do estado nutricional de crianças com menos de 10 anos de idade. Rev. bras. Cienc. Mov., 2(2):7-16, 1988.

3. CARIRI BENIGNA, M.J.; DRICOT, J.; DRICOT D'ANS, C. Crescimento e estado nutricional de crianças de 0-11 anos, Estado da Paraíba (Nordeste brasileiro). Rev. Saúde públ., S. Paulo, 21:480-9, 1987.

4. DESAI, I.D.; GARCIA TAVARES, M.L.; DUTRA DE OLIVEIRA, B. S.;DESAI, M.I.; CEVALLOS ROMERO, L.S.; VICHI, F.L.; DUARTE, F.A.M.; DUTRA DE OLIVEIRA, J.E. Anthropometric and cycloergometric assessment of the nutritional status of the children of agricultural migrant workers in Southern Brazil. Amer. J. clin. Nutr., 34:1925-34, 1981.

5. EVELETH, P.G. \& TANNER, J.M. Worldwide variation in human growth. Cambridge, Engl., Cambridge University Press, 1976.

6. FRISANCHO, A.R. New norms of upper limb fat and muscle areas for assessment of nutritional status. Amer. J. clin. Nutr., 34:2540-5, 1981.

7. GRACEY, M. Normal growth and nutrition. Wld Rev. Nutr. Diet., 49:160-210, 1987.

8. GROSS, R.; STANGE, M.; SOLOMONS, N.W.; OLTERRSDDORF, U.; ESQUIVEL, I.R. The influence of economic deterioration in Brazil on the nutritional status of children in Rio de Janeiro, Brazil. Ecol. Food Nutr., 19:265-79, 1987.

9. HABICHT, J.P.; MARTORELL, R.; YARBROUGH, C.; MALINA, R.M.; KLEIN, R.E. Height and weight standards for preschool children: how relevant are ethnic differences in growth potential? Lancet, 1:611-5, 1974.

10. HAMILL, P.V.V.; DRIZD, T.A.; JOHNSON, C.L.; REED, R.B.; ROCHE, A.F. NCHS growth curves for children birth -18 years. Vital Hith Statist. Ser. $11(165) 1977$.
11. HEGG, R.V. Dobras cutâneas em escolares de sete a onze anos. Rev. Saúde públ., S. Paulo, 12:147-50, 1978.

12. LOHMAN, T.G.; SLAUGHTER, M.H.; BOILEAU, R.A. Description of procedures for the estimation of fat and fat-free body composition of children: a technical manual. Urbana, Univesity of Illinois. Physical Fitness Research Laboratory. Department of Physical Education, 1982.

13. MALINA, R.M. Physical activity and motor development/performance in populations nutritionally at risk. In: Pollitt, E. \& Amante, P., eds., Energy intake and activity. New York, Alan R. Liss, 1984. p.285-302.

14. MARQUES, M.R.; BERQUÓ, E.; YUNES, J.; MARQUES, E. Crescimento de niños brasilentos: peso y altura en relacion con la edad y el sexo y la influencia de factores socioeccnomicos. Washington, D.C., Organiżación Panamericana de la Salud, 1975. (OPAS - Publicación Científica, 309).

15. MATSUDO, V.K.R.; SESSA, M.; TARAPANOFF, A.M.P. Comparação de valores de dobras cutâneas em escolares de áreas industriais e regiōes litorâneas em desenvolvimento. Rev. bras. Cienc. Esporte, 1(3):30-4, 1980

16. MONTEIRO, C.A.; BENICIO, M.H.D'A.; ZUÑIGA, H.P.P.; SZARFARC, S.C. Estudo das condiçðes de saúde das crianças do Município de São Paulo, SP, Brasil, 1984-1985. II - Antropometria nutricional. Rev. Saúde públ., S. Paulo, 20:446-53, 1986.

17. SASR user's guide basics. $5^{\text {th }}$ ed. Gary, N.C., SAS Institute Inc., 1985.

18. SATYANARAYANA, K.; NAIDU, A.N.; RAO, B.S.N. Nutritional deprivation in childhood and body size, activity, and physical work capacity of young boys. Amer. J. clin. Nutr., 32:1769-75, 1979.

19. SOARES, J.; MIGUEL, M.C.; MATSUDO, V.K.R. Desenvolvimento da força de preensão manual em função da idade, sexo, peso e altura em escolares de 7 a 18 anos. Rev. bras. Cienc. Esporte, 2(2):20-4, 1981.

20. TANNER, J.M. Population differences in body size, 
shape, and growth rate. A 1976 view. Arch. Dis. Child., 51:1-2, 1976.

21. TANNER, J.M.; HIERNAUX, J.; JARMAN, S. Growth and physique studies. In: Weiner, J.S. \& Lourie, J.A., comp. Human biology: a guide to field methods. Oxford, England, Blackwell Scientific Publ., 1969. p.1-71. (International Biological Programme, Handbook, n? 9).

22. VICTORA, C.G.; BARROS, F.C.; VAUGHAN, J.P. Epidemiologia da desigualdade. São Paulo, Ed. Hucitec, 1988. p.94-116.

23. WATERLOW, J.C.; BUZINA, R.; KELLER, W.; LANE, J.M.; NICHAMAN, N.Z.; TANNER, J.M. The presentation and use of height and weight data for comparing the nutritional status of groups of children under the age of 10 years. Bull. Wld Hlth Org., 55:489-98, 1977.

24. WORLD HEALTH ORGANIZATION. $A$ growth chart for international use in maternal and child health care: guidelines for primary health care personnel. Geneva, 1978.

25. ZAVALETA, A.N. \& MALINA, R.M. Growth, fatness, and leaness in Mexican-American children. Amer. J. clin. Nutr., 33:2008-20, 1980.

Recebido para publicação em 18/11/88. Reapresentado em $23 / 5 / 89$.

Aprovado para publicação em 26/5/89. 